\title{
Une spiritualité de combat : des syndicalistes chrétiennes en France dans les années 1900-1930
}

Joceline Chabot

\section{(2) OpenEdition}

1 Journals

Édition électronique

URL : https://journals.openedition.org/clio/58

DOI : $10.4000 /$ clio.58

ISSN : 1777-5299

Éditeur

Belin

Édition imprimée

Date de publication : 1 avril 2002

Pagination : 37-54

ISBN : 2-85816-620-X

ISSN : 1252-7017

Référence électronique

Joceline Chabot, « Une spiritualité de combat : des syndicalistes chrétiennes en France dans les années 1900-1930 », Clio. Histoire, femmes et sociétés [En ligne], 15 |2002, mis en ligne le 08 février 2005, consulté le 29 avril 2022. URL : http://journals.openedition.org/clio/58 ; DOI : https://doi.org/ 10.4000/clio.58

Tous droits réservés 


\section{Une spiritualité de combat: des syndicalistes chrétiennes en France dans les années 1900-1930}

Joceline CHABOT

De leur naissance en 1899 à leur dissolution en 1944, les syndicats féminins chrétiens constituent une expérience singulière dans le paysage syndical français ${ }^{1}$. Ces organisations qui regroupent tout au plus quelques dizaines de milliers de membres demeurent marginales par rapport à l'ensemble du mouvement syndical. Cependant, au sein de la Confédération française des travailleurs chrétiens (CFTC) à laquelle elles adhèrent en 1919, elles constituent une force certaine puisque, entre les deux guerres, leurs effectifs représentent entre le quart et le tiers des adhérents à la centrale ${ }^{2}$.

Leur originalité tient, entre autres, à leur mode d'organisation. Pratiquant la non mixité, ces syndicats jouissent d'une large autonomie au sein de la Confédération. Ils profitent du caractère fortement décentralisé de la CFTC pour poursuivre une activité syndicale indépendante structurée autour de l'organisation de services syndicaux, de la formation militante et de cours professionnels. La majorité des syndicats féminins est affiliée à deux "confédérations» féminines qui, sans être en conflit,

1 Il y a peu d'études récentes sur ces organisations, on consultera les travaux de : Bard 1987. Chabot 1998. Ratto, Gautier 1996.

2 Launay 1986.

CLIO, Histoire, Femmes et Sociétés, 2002, 15, pp. 37-54. 
demeurent soucieuses de protéger leur particularisme. La plus ancienne des deux confédérations, l'Union centrale des syndicats professionnels féminins de l'Abbaye, regroupe des syndicats fondés à Paris en 1902 à l'initiative de quelques militantes réunies autour d'une religieuse de la communauté des sœurs de Saint-Vincent de Paul, Sœur Milcent. L'autre fédération naît quelques années plus tard, en 1909, autour de deux militantes catholiques sociales, Andrée Butillard et Aimée Novo. Elle porte le nom de Fédération française des unions de syndicats professionnels féminins. Son siège social est situé boulevard des Capucines à Paris.

Ce mode d'organisation exclusivement féminin a favorisé l'émergence de personnalités fortes qui nous sont révélées à travers les parcours militants. Ces femmes issues en majorité du monde des employés mais également de la classe ouvrière ${ }^{3}$ semblent renoncer à toute vie privée pour se consacrer à leur activité militante. Célibataires, profondément chrétiennes, ces syndicalistes "temps plein" sont animées d'une foi qui, à n'en pas douter, leur donne la force de s'engager et d'agir. Leur itinéraire personnel se confond bien souvent avec celui de l'organisation : «embrayeurs du syndicalisme», pour reprendre une expression chère à Michel Launay, ces militantes, figures de proue du syndicalisme féminin, servent de catalyseur au mouvement syndical que ce soit sur le plan local, régional ou national, et lui permettent non seulement de s'enraciner mais aussi de durer.

Dans le cadre de cet article, nous nous interrogeons sur les motifs qui favorisent l'engagement de ces jeunes femmes chrétiennes dans l'action syndicale. Quels sont les rapports entre l'expérience religieuse et l'engagement syndical ? Nous ne préjugeons pas de la possibilité qu'interviennent d'autres facteurs explicatifs. Cependant, nous estimons qu'il s'agit d'une question essentielle sinon centrale. Notre hypothèse est que ces femmes chrétiennes sont animées par une «spiritualité de combat» ${ }^{4}$ qui, non seu-

3 Pour un portrait sociologique du militantisme féminin chrétien : Chabor $1996: 271$ 284.

4 Nous empruntons cette expression à Dumons 2001, qui l'utilise pour décrire l'action militante des femmes catholiques qui s'engagent dans la Ligue des Femmes Françaises. 
lement, forge leur engagement mais l'enracine dans une culture plus large qui est celle du militantisme catholique social.

\section{Matériaux pour une étude des motivations à l'engagement}

La mesure des rapports entre expérience religieuse et engagement militant n'est pas simple. Un premier problème tient bien sûr aux sources. Contrairement aux sociologues et aux psychologues, les historiens ne peuvent procéder à des enquêtes ou à des entrevues qui leur permettraient d'évaluer, à partir d'un certain nombre de critères, la place de l'expérience religieuse par rapport aux autres facteurs dans les motivations à l'engagement. Il nous faut donc recourir aux témoignages indirects qui nous sont parvenus par le biais des journaux syndicaux, des revues militantes et des biographies.

D'emblée, il faut reconnaître que l'ensemble des sources dont nous disposons demeure bien modeste. Pour la période qui nous intéresse, nous ne possédons aucun récit autobiographique ni aucune biographie crédible et rigoureuse sur les militantes chrétiennes. À notre connaissance, la seule syndiquée qui ait fait l'objet d'un travail biographique est Maria Bardot. Rédigé après son décès par Marguerite Perroy, qui visiblement a connu Maria Bardot, l'ouvrage qui lui est consacré relève davantage de l'hagiographie que du travail scientifique 5

Les matériaux dont nous disposons sont constitués par les récits et les témoignages parus dans les journaux syndicaux durant l'entre-deuxguerres qui relatent les motifs et les circonstances de l'engagement militant. Il s'agit essentiellement des organes des deux grandes fédérations féminines : La Ruche syndicale pour les militantes de l'Union centrale de l'Abbaye et $L a$ Travailleuse pour la Fédération des Capucines ${ }^{6}$. Il faut ajouter à ces deux sources, un certain nombre de journaux régionaux ou locaux comme La femme fonctionnaire, L'Hermine, La Ruche angevine et la

5 Perroy 1931.

6 Le mensuel La Ruche syndicale, dont le format et la périodicité ont varié, a paru à peu près régulièrement de 1902 à 1936. À l'exception de l'année 1916, La Travailleuse a été publiée mensuellement de 1913 à 1936. 
Voix professionnelle. Dans tous les cas, ces récits, qui empruntent l'aspect du témoignage, doivent être utilisés avec prudence car ils constituent bien souvent une forme subtile - et peut-être assez efficace - de publicité pour le recrutement de nouvelles adhérentes. $A$ posteriori, ils servent souvent à justifier l'engagement syndical en fonction des résultats obtenus. À n'en pas douter, les succès seront toujours plus nombreux que les échecs. Audelà de leur caractère quasi publicitaire et de leur forme convenue, une approche critique nous permettra de dégager les procédés argumentaires par lesquels les militantes expliquent et justifient leur engagement.

\section{De la «grâce» militante : expérience religieuse et engagement militant}

La question de l'engagement et du militantisme a suscité de nombreux travaux d'un grand intérêt aussi bien par les problèmes méthodologiques qu'elle soulève que par les lectures possibles qu'elle suggère ${ }^{8}$. Nous retiendrons ici l'exemple de l'engagement jociste qui nous paraît à la fois assez proche de nos préoccupations en même temps que très spécifique par rapport à l'ensemble de la nébuleuse catholique sociale, y compris le syndicalisme chrétien. En étudiant l'histoire de la Jeunesse Ouvrière Chrétienne (JOC) dans l'Ouest, Françoise Richou s'est intéressée, entre autres, aux déterminants socio-culturels de l'idéologie mais aussi de l'adhésion au mouvement. Elle a insisté sur les responsabilités exigeantes imposées aux jeunes militants et sur le processus par lequel un «babitus de combat» leur était inculqué. Selon Richou, il demeure toutefois nécessaire pour beaucoup de militants de recourir à la foi, qu'elle soit de type religieux ou métaphysique, comme soutien à l'action. À ce propos, elle note : "La "grâce" militante, certains jocistes diront la recevoir de Dieu»". Chez les syndicalistes chrétiennes, il n’en est pas autrement. La "grâce» militan-

7 Ces journaux syndicaux possèdent un tirage souvent limité et une parution irrégulière.

8 Parmi une abondante bibliographie, on ne signalera que quelques titres : Dreyfus, Pennetier, Viet-Depaule 1996. Chouvier 1982. Lazar 1998. Wieviorka 1998. Perrot 1998. Michel 1998.

9 Richou $1994: 58$. 
te semble toujours participer de l'expérience religieuse, sinon découler de l'intervention divine. C'est ainsi que s'exprime la vice-présidente de la Fédération du boulevard des Capucines, Mathilde Debray, lorsqu'elle rend compte de l'expérience de la foi dans le processus qui mène à l'engagement :

La syndiquée apôtre [...] a compris le but profond de l'organisation professionnelle, rétablir la justice et la charité dans le monde du travail [...]. C'est une vocation toute spéciale pour laquelle il faut un appel de Dieu [...] semblable à Saint Paul sur le Chemin de Damas. [...] Et avec tout l'ardeur des nouveaux convertis, elle répond : “Oui, Seigneur, je serai apôtre»" ${ }^{10}$.

Ce témoignage ressemble fort aux récits des croyants qui envisagent leur vocation comme étant le fait de l'intervention divine. Selon Debray, le choix de la vie militante relève avant tout d'une expérience mystique. D'après les témoignages publiés dans les journaux syndicaux, nombreuses sont les militantes qui semblent partager ces motivations spirituelles et morales. Pour Alice Vincent, militante de Saint-Étienne, le milieu syndical a représenté "le champ que le Père de famille lui donnait à cultiver" ". Son engagement et son action découlent d'un «appel». Il s'agit bien d'une vocation mais qui s'exerce sur le terrain professionnel. C'est en des termes très semblables que s'exprime Élisa Daniel, vice-présidente du syndicat des ouvrières à domicile, lorsqu'elle raconte comment elle est venue à l'organisation syndicale. "[C'est à la suite d'une semaine de formation] qu'une vraie lumière m'est venue. J'ai mieux compris la réalisation de ces deux mots : Justice et Charités ${ }^{12}$. Selon une autre militante, l'engagement syndical se compare à une croisade pour laquelle la travailleuse a reçu «un mystérieux appel, [sous la forme] d'un trait de lumière décisif» ${ }^{13}$ qui a touché son âme. D'ailleurs, selon l'expression consacrée dans les journaux syndicaux féminins, l'on se fait "propagandiste» - c'est-à-dire militante -

10 La Travailleuse, $\mathrm{n}^{\circ}$ 18, janvier 1917.

11 Le syndicalisme chrétien, 1936.

12 La Travailleuse, $\mathrm{n}^{\circ} 2$, mars 1913.

13 La Travailleuse, $\mathrm{n}^{\circ}$ 42, févr. 1921. 
comme l'on se fait religieuse. Autrement dit, si l'on en croit les témoignages des intéressées, parmi les motivations à l'engagement, l'une des plus importantes, sinon des plus nobles, est «la foi dans l'idée» ${ }^{14}$ qui survient après que la militante ait senti un appel mystérieux ou entrevu une lumière décisive.

Les militantes ont rapporté leurs motivations à l'engagement dans des termes qui relèvent d'un langage spirituel. Selon elles, c'est la volonté divine qui guide leur choix, elle n'ont fait que s'y conformer. À l'instar de la religieuse, la militante répond à un «appel», elle possède la "vocation» mais une vocation dite sociale. Comme l'écrit une militante du boulevard des Capucines, l'engagement syndical "c'est une mission que chacune de nous a reçue de Dieu, une vocation dont dépend notre grandeur et notre récompense futures»" ${ }^{15}$. Elles livrent un combat pour la justice et la charité et elles entendent contribuer à la rechristianisation de la classe ouvrière. En ce sens, l'itinéraire de la militante chrétienne ressemble à une trajectoire puisqu'il actualise dans les circonstances propres à l'engagement les formes virtuelles d'une vocation intime.

À lire les témoignages, il n'y a pas de parcours aléatoire pour la militante chrétienne car le récit de son engagement est celui d'une vocation. $S$ 'il faut s'interroger sur ces parcours qui semblent presque trop «normaux», presque sans hasard, illustrant en quelque sorte ce qui ressemble à un appel du destin et qui, ne l'oublions pas, ne se donnent à lire que dans une reconstruction a posteriori, il ne nous appartient pas de juger de l'intensité ou de la profondeur de la foi qu'ils expriment.

\section{Pèlerinages, emblèmes, devises et insignes : les manifestations de la foi et la pratique syndicale}

L'expérience religieuse ne peut se résumer à la seule "grâce divine». Comme le soulignait René Lemarquis dans un texte portant sur le religieux et le militantisme, cette expérience emprunte diverses formes : elle est liée à certains rites ainsi qu'à la croyance en une valeur absolue, elle se

14 L'Hermine, $n^{\circ}$ 6, déc. 1927.

15 La Travailleuse, $\mathrm{n}^{\circ}$ 8, nov. 1913. 
reconnaît dans l'appartenance à une communauté et procède d'aspects comme le sentiment du sacré ou le mysticisme ${ }^{16}$. Comment mesurer cette expérience ou plutôt son impact, sa relation avec le militantisme ? Lemarquis suggère certaines pistes qui pourraient nous permettre de prendre la mesure de cette relation et non pas de l'expérience elle-même ${ }^{17}$. Il faudrait, selon lui, tenir compte de la profondeur de la foi religieuse mais aussi des expériences de l'enfance, des rencontres et des influences. Bref, d'un ensemble de facteurs qui sont rarement perçus par les acteurs comme essentiels dans le processus qui mène à l'engagement.

Il est difficile de juger de la sincérité de la foi religieuse des militantes chrétiennes à partir des témoignages dont nous disposons. Par ailleurs, rien ne nous permet de les remettre en question. En revanche, il est plus aisé de mesurer les pratiques liées à la foi dans le cadre ou parallèlement à l'activité syndicale. L'intérêt de cette exploration nous semble résider dans le fait qu'à partir des pratiques et des comportements, il est peut-être possible d'atteindre une connaissance des consciences, là où, selon René Rémond, réside le lieu par excellence du religieux ${ }^{18}$. En effet, on peut estimer que l'adhésion des militantes à certaines pratiques représente un indice sérieux de leur foi. Il ne s'agit donc pas de traquer quelques manifestations insolites $\mathrm{du}$ fait religieux dans la pratique et l'action militantes des syndicalistes chrétiennes mais, au contraire, d'être sensible à l'appropriation et à l'intégration des signes manifestes de la foi dans le cadre de la vie militante. Par exemple, nous savons que le congrès annuel des fédérations féminines est toujours précédé ou suivi d'une messe. Pour les militantes, cette manifestation de leur foi s'intègre parfaitement à leur vision de l'engagement syndical fondée sur l'union fraternelle dans un esprit chrétien. C'est ainsi que s'exprime l'une d'elles lors de la réunion du conseil syndi-

16 Lemarquis $1996: 285$.

17 Ce n'est pas notre objet d'étude. Certains contestent la possibilité pour les historiens à partir de leur méthode de connaître cette expérience. Selon Dupront 1987, seule l'approche phénoménologique peut permettre d'atteindre à une connaissance de l'expérience religieuse vécue. Dans cette perspective, cette expérience serait irréductible à la méthode historique.

18 Rémond 1988 : 93-107. 
cal des employées :

Nous ne sommes pas groupées seulement pour soutenir nos intérêts temporels : nous devons nous montrer chrétiennes dans toutes les circonstances de la vie; le lien étroit qui doit nous unir, c'est l'amour chrétien ${ }^{19}$.

De la même manière, les syndicats de l'Abbaye et plus tard, ceux des Capucines, organisent pour leurs membres un pèlerinage annuel au SacréCœur de Montmartre. Cette pratique n'est pas individuelle mais bel et bien partagée par la collectivité alors que les syndiquées assistent à une messe spéciale en leur honneur ${ }^{20}$. Le but visé est double : il s'agit de manifester collectivement sa foi et de permettre aux membres du syndicat de mieux se connaître «en vue de développer un véritable esprit de famille»" ${ }^{21}$. En revanche, si l'adhésion des militantes du boulevard des Capucines à la Confrérie Notre Dame du Travail n'est pas liée à l'activité syndicale «elle en est le complément, le couronnement, en quelque sorte» ${ }^{22}$. L'adhésion des syndiquées à une confrérie religieuse poursuit le même objectif que l'assistance à la messe lors du pèlerinage annuel des syndicats de l'Abbaye : "Elle n'arrête ou ne diminue en rien l'action propre du Syndicat : elle ne fait que resserrer le lien qui unit les membres" ${ }^{23}$. C'est la Confrérie qui, par exemple, offre une messe aux syndiquées qui se marient ou qui décèdent. Elle organise les visites aux malades. Elle possède une chorale qui anime les réunions du cercle d'études. Nous retrouvons ici des pratiques traditionnelles de la sociabilité chrétienne qu'elle soit féminine ou masculine. À n'en pas douter, les militantes estiment que ces pratiques forgent leur engagement syndical parce qu'elles fondent leur communauté.

Par ailleurs, les syndiquées n'hésitent pas à s'approprier certaines manifestations de la culture ouvrière et à les transformer de façon à ce qu'elles

19 La Ruche syndicale, 3e année, $n^{\circ}$ 9, oct.-déc. 1904.

20 La Ruche syndicale, Ge année, $n^{\circ} 27,15$ janv. 1908 ; 26e année, nov. 1928.

21 La Ruche syndicale, 5e année, $n^{\circ} 18$, janv.-février 1907.

22 La Travailleuse, 3 e année, $\mathrm{n}^{\circ} 20$, août 1917. La Confrérie aurait été fondée à Lyon par une catholique sociale, Madeleine Carsignol. En 1917, elle regrouperait une centaine de membres qui ne sont sans doute pas toutes liées au mouvement syndical.

23 La Travailleuse, $3 e$ année, $n^{\circ} 20$, août 1917. 
s'intègrent dans la sphère particulière de leurs pratiques chrétiennes et militantes. C'est ainsi que si on célèbre volontiers le ler mai chez les syndicalistes chrétiennes, c'est à l'archevêché de Paris que se tient la fête. C'est l'occasion pour les représentantes des syndicats de rencontrer l'archevêque, de recevoir sa bénédiction et d'écouter ses bons conseil ${ }^{24}$. Les fêtes syndicales organisées lors d'un anniversaire ou d'un événement spécial constituent un moment privilégié pour manifester sa foi dans le cadre d'une sociabilité militante. Par exemple, l'inauguration de nouveaux locaux commence toujours par la bénédiction des lieux par un prêtre avant que les syndiquées ne s'adonnent à des réjouissances plus profanes, puisqu'aussi bien l'on y danse et l'on y chante. Un exemple nous en est donné lorsqu'en 1917 la Fédération des syndicats féminins s'installe à son nouveau siège social. La cérémonie se déroule un dimanche et c'est un vicaire de la Madeleine, l'abbé Hertzig, qui est sollicité pour la bénédiction. La journée se poursuit par une "causerie syndicale" et se termine, après le goûter, par des chants syndicaux ${ }^{25}$. Si les militantes apprécient le caractère solennel que revêt la bénédiction ou la simple parole du prêtre lors des réunions syndicales, cette pratique demeure circonscrite dans un espace/temps précis, soit au début ou à la fin de la journée, parfois lors du repas du midi, et, généralement, lors des fêtes et des événements particuliers. Mais il ne saurait être question d'inviter un religieux lors des réunions syndicales ordinaires. C'est que la confusion des genres est toujours possible et le risque est grand pour les syndicats de perdre leur caractère professionnel et d'être assimilés à une ouvre sociale catholique. Danger permanent, puisqu'en 1929 lors de l'assemblée annuelle des Syndicats professionnels féminins versaillais affiliés à l'Union centrale de l'Abbaye, Jeanne Graff, figure de proue du syndicalisme féminin, met en garde les syndiquées : "il ne faut pas confondre le Syndicat avec les œuvres, même professionnelles, auxquelles on peut appartenir ${ }^{26}$.

24 Cette cérémonie qui est rapportée en détail dans le journal des syndicats de l'Abbaye n'a pas duré au-delà des années 1910. La Ruche syndicale, 3e année, mai-juin 1904 ; 4 e année, mai-juin 1905.

25 La Travailleuse, 3e année, $\mathrm{n}^{\circ} 18$, janv. 1917.

26 La Ruche syndicale, 27e année, mars 1929. 
C'est dans les premières années de leur existence, en ayant recours à une symbolique très marquée, que les syndicats de l'Abbaye vont afficher le plus fortement leur caractère confessionnel. Les insignes et les devises dont ils se dotent participent de cette affirmation ${ }^{27}$. C'est ainsi que lors des réunions et des fêtes, les syndiquées de l'Abbaye arborent fièrement leur insigne qui est constitué d'une médaille de Jeanne d'Arc et d'un ruban de couleur. De la même manière, les premières organisations qui donnent naissance à l'Union centrale choisissent des devises empruntées à la religion chrétienne : "Aimez-vous les unes les autres», "Vive Labeur !» (attribuée à Jeanne d'Arc). Dans le palmarès des figures féminines issues du répertoire catholique, on retrouve celle de Jeanne d'Arc qui demeure sans conteste la plus populaire. À preuve, le nombre important de prières, récits, poèmes qui lui sont consacrés et dont on retrouve les traces dans la presse syndicale ${ }^{28}$.

Un dernier exemple est constitué par l'emblème syndical illustrant la première page de La Ruche syndicale qui reprend les mêmes symboles: les devises sont inscrites sur des rubans qui entourent une ruche bourdonnante placée à l'ombre de la Croix. Selon les militantes, l'ensemble de ces représentations incarne "la charité chrétienne, la dignité du travail, la solidarité fraternelle», elles "conviennent [...] à merveille à des femmes chrétiennes qui, obligées à la lutte pour la vie, l'acceptent avec courage, avec joie et, cherchent à s'aider les unes les autres pour alléger leur tâche, la rendre plus profitable et meilleure ! ${ }^{29}$

Ces quelques exemples témoignent d'une appropriation des signes, des rites et des pratiques de la foi et de leur intégration dans le cadre de l'ac-

27 À notre connaissance, la Fédération du boulevard des Capucines ne semble pas avoir adopté ce mode de distinction et de reconnaissance sauf en ce qui concerne la devise syndicale : "Nous aimer, nous aider, nous défendre", et encore, celle-ci n'est pas directement tirée du lexique chrétien. En revanche, avec la devise du syndicat des jeunes filles de Nantes affilié à l'Abbaye : "Plutôt la mort que la souillure" on est au couur du registre moral et religieux.

28 On retrouve plusieurs exemples de cette dévotion à Jeanne d'Arc dans La Ruche syndicale mais aussi dans L'Hermine, le journal des jeunes syndiquées de Nantes.

29 La Ruche syndicale, $\mathrm{n}^{\circ}$ 1, oct.-déc. 1902, p. 2. 
tivité syndicale. Chez les militantes chrétiennes, la célébration de la foi s'inscrit au sein même de la vie syndicale. Celle-ci ne peut être dissociée d'une pratique religieuse qui forge l'engagement. Mais pour autant l'expérience religieuse fonde-t-elle l'engagement ?

\section{Des motivations à l'engagement ou comment l'esprit syndical vient aux chrétiennes}

Pour une meilleure connaissance des rapports entre l'expérience religieuse et l'engagement militant, il nous semble important d'introduire deux éléments explicatifs : les expériences de jeunesse et les influences.

En 1909, Maria Bardot, jeune ouvrière de la couture, fréquente le patronage Sainte-Agnès à Paris où, selon sa biographe, elle assiste à une conférence sur les syndicats chrétiens. Comme de nombreux catholiques, Maria Bardot ignore tout de ce mouvement syndical. Mais à compter de cette date et jusqu'à la fin de sa vie, son itinéraire est tout tracé et se confond avec son engagement dans les organisations féminines. A posteriori, Maria Bardot expliquera à sa biographe les motifs de son adhésion au syndicat :

La compréhension de ma responsabilité en tant qu'ouvrière : parce que catholique, je n'avais pas le droit de me désintéresser de cette organisation du monde du travail, basée sur les principes de la doctrine catholique. Le désir de lutter contre les principes socialistes qui perdent les âmes, le désir de travailler au règne de Dieu dans les âmes de ma famille ouvrière ${ }^{30}$.

Cette citation appelle trois remarques. D'abord, cette confession recueillie par sa biographe des années plus tard ressemble trop à une leçon bien apprise pour lui accorder un crédit exagéré. En effet, tout y est, la vision claire de la mission à venir, le désir de lutter contre les socialistes qui perdent les âmes, la nécessité de rechristianiser le monde ouvrier. Ensuite, à travers cette réflexion, Maria Bardot exprime l'antériorité de sa foi par rapport à son engagement. René Rémond a souligné l'importance de ce phénomène pour comprendre la mentalité religieuse : «[...] s'il est

30 Perroy 1931 : chap. 2. 
une certitude [c'est que] la croyance est antérieure aux comportements et aux engagements ${ }^{31}$.

Troisième remarque, ce témoignage n'est pas dépourvu de renseignements, d'indices par rapport à ses expériences de jeunesse et aux influences qu'elle a reçues. Mais il est nécessaire de le replacer dans un contexte plus large. Nous apprenons ainsi que c'est par le biais d'un patronage catholique que la jeune fille connaît l'existence des organisations féminines. Une expérience partagée par Madeleine Tribolati, militante de la Fédération du boulevard des Capucines et future vice-présidente de la CFTC, qui fréquente l'école laïque et dont le milieu familial s'avère indifférent à la religion. Elle reçoit l'essentiel de son éducation religieuse au patronage catholique. C'est également "au patro" que, pour la première fois, elle entend parler des syndicats féminins ${ }^{32}$. On peut citer aussi l'exemple d'Anna Schiff, militante de la première heure dans la région de Nancy : l'aumônier du patronage de la paroisse de Notre-Dame-duRosaire - dans le $\mathrm{XIV}^{\mathrm{c}}$ arrondissement de Paris - l'oriente vers la Fédération du boulevard des Capucines. Contrairement à Tribolati, Schiff provient d'une famille très catholique et reçoit une éducation religieuse. Quant à Marie-Louise Danguy, une des propagandistes les plus actives des syndicats féminins et de la CFTC, elle s'occupait d'un patronage de jeunes filles jusqu'à 1919, alors qu'elle fait la connaissance d'Andrée Butillard, l'une des fondatrices des syndicats féminins. Cette rencontre déterminera son engagement dans le syndicalisme chrétien ${ }^{33}$.

Combien parmi les militantes syndicalistes chrétiennes ont ainsi fréquenté les "patros» comme on les appelle familièrement ? Il est impossible de répondre précisément à cette question, mais il n'est pas exagéré de penser qu'elles sont fort nombreuses. Les syndicats féminins considèrent ce milieu propice au recrutement et ne lésinent pas sur la propagande afin d'y faire connaître leurs organisations ${ }^{34}$. Dans ce contexte, il n'est guère

31 Rémond $1988: 107$.

32 C'est ce qu'elle nous confiait lors d'une entrevue réalisée à Paris en 1990.

33 Pascal $1982: 30$.

34 Les syndicats masculins possèdent une politique similaire. Au congrès de la CFTC, en 1930 , Gaston Tessier insiste pour que toutes les unions et les syndicats locaux demeu- 
surprenant de retrouver ces structures comme l'une des principales voies à l'origine de l'adhésion et peut-être de l'engagement dans le milieu syndical chrétien. Le patronage peut également se révéler un concurrent pour le syndicat. En effet, les patronages assurent souvent les mêmes services pratiques que les organisations syndicales. Il devient alors difficile d'y intéresser les jeunes filles déjà bien servies par le "patro" ${ }^{35}$.

Mais les origines sociales et familiales des militantes préexistent à ces expériences. C'est au sein de la famille que les jeunes enfants expérimentent les premiers outils de socialisation en même tant qu'ils reçoivent les bases de leur éducation. Dans cette perspective et compte tenu de l'importance de ce facteur, ne doit-on pas se demander combien parmi les militantes chrétiennes proviennent de familles catholiques? Malheureusement, il est difficile de répondre à cette question car trop souvent le milieu familial des militantes demeure inconnu. Les quelques informations dont nous disposons sont trop fragmentaires pour que nous puissions en tirer des conclusions pertinentes. En effet, pour une Maria Bardot et une Marie-Louise Danguy élevées au sein d'une famille pratiquante, nous retrouvons une Madeleine Tribolati et une Simone Troisgros, militantes à la Fédération du boulevard des Capucines, dont les parents sans être hostiles à l'Église, demeurent cependant indifférents à la religion. Au demeurant, l'engagement militant n'est-il pas la résultante de motivations complexes forgées au fil d'un parcours marqué par les événements et les rencontres ? Que la croyance soit antérieure à l'engagement voilà qui nous semble confirmé pour bon nombre de militantes chrétiennes. Pour autant, cela n'explique ni la nature de cet engagement, ni les voies par lesquelles il advient. Dès lors, s'il y a un lien et peut-être une relation de causalité entre l'expérience religieuse et l'engagement militant pour les syndicalistes chrétiennes, c'est à partir des influences et des rencontres qu'il faut le rechercher.

rent en contact étroit avec les organisations et les œuvres catholiques. Il suggère de consolider cette liaison grâce à l'établissement de sections fonctionnant au sein même des cuvres. "Le $11^{c}$ congrès de la CFTC", La Travailleuse, n 128 , juill.-août, 1930.

35 Sur les rapports parfois difficiles entre les deux mouvements on peut se reporter au témoignage d'une dirigeante de section paru dans La Travailleuse, $\mathrm{n}^{\circ} 143$ et 144 , 
Un fait nous paraît essentiel : à un moment ou à un autre, il y a dans l'itinéraire des militantes chrétiennes une rencontre. Nous comprenons le terme de rencontre dans sa double acception : d'une part les circonstances parfois fortuites qui placent une personne dans une situation propice à l'action. D'autre part, la volonté exprimée et manifeste d'aller au-devant d'une personne ou d'un idéal. Dans cette seconde perspective, le mot "rencontre" signifie l'action de s'engager pour une cause, de livrer combat dans le but d'assurer le triomphe de ses idées. Pour plusieurs militantes, il semble qu'il y ait dans leur itinéraire la présence de ces deux phénomènes : à savoir le fait du hasard et le principe de finalité, là où s'engage la lutte pour l'idéal motivée par une "spiritualité de combat».

Il en est ainsi pour Marie-Louise Danguy qui sent en elle l'appel de Dieu, la vocation, et qui pense alors se faire religieuse. $\grave{A}$ la suite des événements entourant la grève des banques, en 1919, et d'une rencontre avec Andrée Butillard, elle opte pour l'engagement syndical. Quant à Maria Bardot, sa biographe raconte les événements entourant son choix de vie en insistant sur le doute qui assaille la jeune fille au moment où elle décide d'abandonner la possibilité de se faire religieuse cloîtrée, au profit d'une activité plus profane dans un milieu moins confiné. Sur les conseils d'une sœur du Cénacle et après la rencontre d'un prêtre, partisan des syndicats féminins, Maria Bardot opte finalement pour l'action professionnelle et syndicale. On pourrait également mentionner Eugénie Pradel, Marie-Camille Pelevillain, Eugénie Beeckmans dont l'adhésion aux syndicats de l'Abbaye en 1902 est motivée par leur rencontre avec Sœur Milcent. Quant à Simone Troisgros, c'est la personnalité rayonnante de Marguerite Martin, une propagandiste active de la Fédération du boulevard des Capucines, qui la convainc de joindre les rangs de l'organisation chrétienne. Qu'il s'agisse d'un prêtre, d'une religieuse ou d'une laïque engagée dans l'action sociale catholique, ces contacts jouent un rôle important, sinon essentiel dans l'engagement militant. Ils permettent à ces jeunes femmes chrétiennes d'inscrire dans une réalité qui est la leur, celle du monde du travail, le sens d'une «vocation». C'est le point focal

février et mars 1932. 
qui met en rapport l'expérience religieuse et le besoin d'agir pour la réalisation d'un idéal. Le cadre dans lequel se réalise cet engagement sera finalement celui du mouvement syndical.

Les militantes syndicalistes chrétiennes ont vécu leur engagement comme une forme d'apostolat au service d'un idéal. Elles n'hésitent pas à proclamer haut et fort leur foi en s'appropriant et en intégrant dans l'action syndicale les manifestions les plus sensibles de la culture catholique. La mise en œuvre de ces pratiques forge en quelque sorte l'engagement autour de l'affirmation d'une foi militante dans le cadre particulier de l'organisation professionnelle. Toutefois, on aurait tort d'assimiler la militante syndicaliste chrétienne à la «dame d'œuvre», figure dominante des milieux catholiques féminins, même si sa version contemporaine - telle qu'elle émerge dans le cadre d'une action sociale comme celle menée par Andrée Butillard ${ }^{36}$ - permet de la distinguer de la dame patronnesse plus traditionnelle. Les syndicats féminins chrétiens ne relèvent pas des œuvres catholiques, ils sont indépendants des mouvements d'action catholique même si, à l'occasion, des liens se tissent entre ces différentes organisations autour d'un événement ou d'une cause. Si les militantes syndicalistes estiment faire ouvre d'apostolat, il s'agit d'un apostolat social qui non seulement pose la question de l'amélioration des conditions de vie des travailleuses mais surtout cherche à y apporter des solutions concrètes par l'action revendicative dans le cadre de l'organisation syndicale et sur le terrain professionnel. Il faut également rappeler que ces militantes sont issues des milieux dans lesquels elles exercent cet apostolat social. Elles sont travailleuses, employées pour la majorité mais aussi pour bon nombre d'entre elles, ouvrières.

L'engagement des syndicalistes chrétiennes differe également de celui des militantes jocistes même si, les unes et les autres, entendent participer à la rechristianisation de la classe ouvrière. Le jocisme est un mouvement autonome et non clérical mais apostolique et missionnaire ${ }^{37}$. L'engagement jociste est d'abord et avant tout témoignage du Christ dans

36 Rollet 1960.

37 Pierrard 1984 : 529. 
le milieu de vie. Si les manifestations collectives et les revendications sociales participent de l'action jociste, le témoignage - élément essentiel de la spiritualité jociste - est d'abord individuel et, en ce sens, l'aspect social de l'engagement jociste relève de l'influence personnelle ${ }^{38}$. Les militantes syndicalistes chrétiennes agissent dans le monde du travail qui conditionne leur action revendicative autour de préoccupations essentiellement collectives et réformistes ${ }^{39}$. L'engagement n’est pas vécu de la même manière pour les jocistes et les syndicalistes et, surtout, il n'est pas vécu à partir des mêmes cadres sociologique et institutionnel.

Si l'engagement des militantes chrétiennes comporte des éléments qui permettent de marquer sa spécificité, il nous semble également posséder un certain nombre de composantes largement partagées par "l'esprit militant». La tâche première de la militante au sein de l'organisation professionnelle est de «servir». Elle est au service d'une cause, d'un idéal. Se sacrifier, se donner, servir, l'un ou l'autre de ces termes revient systématiquement dans tous les articles consacrés au militantisme. Autour de ces notions, nous distinguons ce qui fonde l'engagement des militantes syndicalistes chrétiennes et que nous avons identifié comme une "spiritualité de combat». Or, ces notions sont partagées par de nombreux militants chrétiens. Françoise Richou les reconnaît chez les militants jocistes qui intériorisent les normes de l'expérience religieuse en «associant vie spirituelle et action $»^{40}$. Bruno Dumons, quant à lui, les attribue aux militantes qui créent, en 1901, la Ligue des Femmes Françaises en insistant sur leur esprit missionnaire façonné par le catholicisme intransigeant et sur leur engagement vécu comme une expiation des péchés du monde ${ }^{41}$. Il y aurait là une forme d'engagement qui favoriserait, au-delà des particularités liées au milieu et des contingences du temps, l'éclosion d'un esprit, d'une mentalité militante. Un engagement de type existentiel ${ }^{42}$ qui imprègne toute

38 Launay 1985 : 223-231. Richou 1994 : 51-82.

39 Launay 1984 : 59-63.

40 Richou 1994 : 56.

41 Dumons 2001.

42 Nous empruntons ce concept à Annie Kriegel qui l'utilise pour distinguer les degrés d'adhésion au Parti communiste français. Lazar $1998: 36$. 
la vie sociale du militant et qui, chez les chrétiens, conjugue la foi et l'action. À n'en pas douter, c'est à travers cette spiritualité combattante que l'expérience religieuse participe de l'engagement militant chez les syndicalistes chrétiennes.

\section{Bibliographie}

BARD Christine, 1987, Les femmes et la CFTC à travers le "Nord social» 1920-1936, mémoire de maîtrise, Université de Lille.

CHABOT Joceline, 1998, Le syndicalisme féminin chrétien en France de 1899 à 1944 : pratiques et discours d'une culture féminine, thèse pour le doctorat, Université de Paris 8.

-, 1996, "Les femmes syndicalistes chrétiennes dans le Maitron», in Michel Dreyfus, Claude Pennetier, Nathalie Viet-Depaule (dir.), La part des militants, Paris, Éditions de l'Atelier, pp. 271-283.

CHOUVIER Bernard, 1982, Militance et inconscient, les fondements psychologiques, Lyon, PUL.

DUMONS Bruno, 2001, «Entrer en politique pour accomplir sa vie. Itinéraire d'une bourgeoise catholique lyonnaise : Jeanne Lestra et la Ligue des Femmes Françaises (1901-1914)», communication au colloque Un siècle de vie associative : quelles opportunités pour les Femmes?, Paris.

DUPRONT Alphonse, 1987, Du sacré. Croisades et pèlerinage. Images et langages, Paris, Gallimard.

LAUNAY Michel, 1986, La CFTC, origines et développement 1919-1940, Paris, Publications de la Sorbonne.

-, 1985, "Réflexions sur les origines de la JOC», in Gérard Cholvy (dir.), Mouvements de jeunesse chrétiens et juifs. Sociabilité juvénile dans un cadre européen, 1789-1968, Paris, Éditions du Cerf.

-, 1984, "La J.O.C. dans son premier développement», in Michel Launay, Pierre Pierrard, Rolande Trempé, J.O.C. : Regards d'historiens, Paris, Éditions Ouvrières, 1984.

LAZAR Marc, 1998, "Le Parti et le don de soi", Vingtième siècle. Revue d'histoire, 60, pp. 35-42. 
LEMARQUIS René, 1996, "Le religieux et le militantisme dans le Dictionnaire biographique du mouvement ouvrier français, in Michel Dreyfus, Claude Pennetier, Nathalie Viet-Depaule (dir.), La part des militants, Paris, Éditions de l'Atelier, pp. 285-311.

MICHEL Joël, 1998, "Le syndicalisme, un horizon sans grandeur ?», Vingtième siècle. Revue d'histoire, 60, pp. 27-34.

PASCAL Henri, 1982, Monographie sur l'École Normale sociale 19111960, Paris, texte dactylographié.

PERROT Michelle, 1998, "La cause du peuple», Vingtième siècle. Revue d'histoire, 60, pp. 4-13.

PERROY Marguerite, 1931, Une ouvrière apôtre social : Maria Bardot, Paris, Éditions Spes.

PIERRARD Pierre, 1984, L'Église et les ouvriers en France (1840-1940), Paris, Hachette.

RATTO Martine, GAUTIER Andrée, 1996, "Les syndicats libres féminins de l'Isère, 1906-1936", CLIO, Histoire, Femmes et Sociétés, 3, pp. 117-139.

RÉMOND René, 1988, "L'histoire religieuse de la France au XXe siècle», Vingtième siècle. Revue d'histoire, 17, pp. 93-107.

RICHOU Françoise, 1994, «Apprendre à combattre : l'engagement dans la Jeunesse Ouvrière Chrétienne (1927-1987)», Le Mouvement social, 168, pp. 51-83.

ROLLET Henri, 1960, Andrée Butillard et le féminisme chrétien, Paris, Édition Spes.

WIEVIORKA Olivier, 1998, «À la recherche de l'engagement (19401944)», Vingtième siècle. Revue d'histoire, 60, pp. 58-70. 